\title{
Role of the Posterior Parietal Cortex in Spatial Hearing
}

\author{
Jörg Lewald, ${ }^{1,2}$ Henrik Foltys, ${ }^{3}$ and Rudolf Töpper ${ }^{3}$ \\ 1/nstitute for Occupational Physiology, D-44139 Dortmund, Germany, ${ }^{2}$ Department of Cognitive and Environmental \\ Psychology, Ruhr University, D-44780 Bochum, Germany, and '3Department of Neurology, University Hospital of Aachen, \\ D-52074 Aachen, Germany
}

The human posterior parietal cortex (PPC) is well known to be involved in various functions of multisensory spatial perception. However, the specific role of the PPC in hearing has, up to now, remained unclear. To allow more reliable conclusions to be drawn on this issue, we have used repetitive transcranial magnetic stimulation in healthy subjects. Focal stimulation of the PPC induced a systematic shift in the lateralization of interaural time differences (ITDs, a main cue for auditory azimuth), whereas the acuity of ITD discrimination was unaffected. We propose that the PPC is specifically involved in relating azimuthal angles of sound to the body coordinates and is part of a "where" stream in cortical processing of auditory information.

Key words: posterior parietal cortex; repetitive transcranial magnetic stimulation; interaural time differences; sound localization; space perception; psychophysics; human
Unlike visual spatial perception, spatial hearing is based not on the representation of sensory stimuli on a receptor epithelium but on the evaluation of specific cues of the sound received by the two ears. In particular, interaural differences in time of arrival and level as well as spectral cues are used to derive azimuthal and elevational coordinates of the position of a sound source with respect to the ears. Neural correlates of these basic processes, synthesizing representations of acoustic space, have been located in the subcortical auditory pathway up to the primary auditory cortex (Masterton, 1992; Middlebrooks, 2000). The role of brain regions beyond the primary auditory cortex in higher-order perceptual processes of spatial hearing is, however, poorly understood. Anatomical and physiological results obtained recently in the monkey have suggested that the spatial component of auditory information is processed within a "dorsolateral stream", which includes the caudal superior temporal cortex, posterior parietal cortex (PPC), and dorsolateral prefrontal cortex, and is anatomically distinct from a "ventrolateral stream", processing nonspatial acoustic features (Rauschecker, 1998; Romanski et al., 1999; Rauschecker and Tian, 2000). This seems to be analogous to the widely held view of parietal "where" and temporal "what" processing streams for transmission of visual spatial and object information, respectively (Mishkin et al., 1983). Starting from this hypothesis, the present study focused on the functional characterization of the human PPC in auditory localization. For this purpose, we aimed to selectively affect neural processing in this area by repetitive transcranial magnetic stimulation (rTMS). With this technique, high-current pulses, generated in a coil of wire placed above the scalp, produce magnetic fields that induce electric fields (Hallett, 2000; Walsh and Cowey, 2000). The repetitive electric-field changes during rTMS stimulate neurons of

Received Sept. 21, 2001; revised Nov. 5, 2001; accepted Nov. 27, 2001.

This work was supported by Grants Gu 261/7-1, Eh 91/4-2, and To 190/2-3 from the Deutsche Forschungsgemeinschaft. We thank P. Dillmann for preparing the software and parts of the electronic equipment and W. H. Ehrenstein, S. J. Fellows, and H.-O. Karnath for valuable comments on the manuscript.

Correspondence should be addressed to Jörg Lewald, Fakultät für Psychologie, Ruhr-Universität, D-44780 Bochum, Germany. E-mail: joerg.lewald@ruhr-unibochum.de.

Copyright (ㄷ) 2002 Society for Neuroscience $\quad 0270-6474 / 02 / 220001-05 \$ 15.00 / 0$ the underlying cortical surface in an uncoordinated manner, thus interrupting normal brain activity. Transient alterations of the activity of the stimulated area have been shown to persist for several minutes after application of rTMS (Pascual-Leone et al., 1994; Chen et al., 1997; Mottaghy et al., 1999; Töpper et al., 1999). In the present study, sound lateralization was tested during this rTMS after-effect to minimize interference between the auditory stimuli used and the sound pulses accompanying rTMS pulses, as well as those between magnetic stimulation and peripheral hearing functions.

\section{MATERIALS AND METHODS}

Ten right-handed male subjects (27-36 years of age) participated in this study. The experimental room was silent and darkened, with only a fixation target (dim light spot) straight ahead of the subject. The subject was seated, with the head fixed in a straight-ahead position by a stabilizing rest for the occiput. Dichotic pure-tone pulses (frequency, $1 \mathrm{kHz}$; sound pressure level, $70 \mathrm{~dB}$; plateau time, $30 \mathrm{msec}$; rise/fall time, 10 $\mathrm{msec}$ ) were presented via insert earphones (ER-1; Etymotic Research Co., Elk Grove Village, IL) that were worn in combination with hearingprotection earmuffs. The interaural time difference (ITD) of the sound stimulus was varied between trials following a quasi-random order over a range from $-112.5 \mu \mathrm{sec}$ (sound leading at the left ear) to $+112.5 \mu \mathrm{sec}$ (leading at the right ear), in steps of $22.5 \mu \mathrm{sec}$. Within $1 \mathrm{sec}$ after stimulus presentation, subjects were required to indicate the perceived intracranial position of the sound with respect to the median plane of the head by pressing a "left" or "right" key. Sound stimuli were presented with intervals of $3 \mathrm{sec}$. After a few practice trials, two subsequent blocks, each composed of 220 trials (equaling $11 \mathrm{~min}$ ), were conducted as the prerTMS reference condition.

This article is published in The Journal of Neuroscience, Rapid Communications Section, which publishes brief, peerreviewed papers online, not in print. Rapid Communications are posted online approximately one month earlier than they would appear if printed. They are listed in the Table of Contents of the next open issue of JNeurosci. Cite this article as: JNeurosci, 2002, 22:207 (1-5). The publication date is the date of posting online at www.jneurosci.org.

http://www.jneurosci.org/cgi/content/full/6072 
rTMS was applied with a magnetic stimulator (Magstim Rapid Stimulator; Magstim Co., Spring Gardens, UK) connected with a figure-eight coil (outer diameter, $9 \mathrm{~cm}$ ). The magnetic stimulator was triggered by an external device (Master 8 Trigger unit; AMPI, Jerusalem, Israel) with a frequency of $1 \mathrm{~Hz}$ for $10 \mathrm{~min}$ (total number of stimuli was 600). The intensity of magnetic stimulation was set to $60 \%$ of the stimulator output (maximum output, 2.2 tesla). All stimulation parameters were in accordance with the safety guidelines for repetitive magnetic stimulation (Wassermann, 1998). rTMS was delivered to the posterior parietal cortex of the left or right hemisphere, and the stimulation sites P3 and P4 were determined according to the 10-20 EEG system. Immediately after rTMS application, two blocks of the psychophysical task were completed as for the reference condition. After a rest of at least $2 \mathrm{~h}$, this procedure was repeated with rTMS of the subject's other hemisphere. For each block, the subject's judgments were determined as a function of ITD and data were fitted to the following sigmoid equation: $f=100 /[1+$ $\left.e^{-k(\mathrm{ITD}-\mathrm{AMP})}\right]$, where $f$ is the frequency of judgments "right", given as a percentage; the "auditory median plane" (AMP) is the ITD for which $f$ is $50 \%$; $k$ is the slope of the function at $50 \%$; and $e$ is the base of the natural logarithm (compare Fig. $1 A$ ). The fit was significant in each case (coefficient of determination: $r^{2}>0.92 ; p<0.0001$ ). The values $k$ and $r^{2}$ obtained from the fit were used here as measures for the subject's acuity of sound lateralization, whereas the AMP values indicated systematic shifts in lateralization. AMPs were normalized such that values obtained with the pre-rTMS blocks 1 and 2 were assigned $0 \mu$ sec. Negative displacements of the AMP with the post-rTMS blocks 1 and 2 thus indicate shifts in auditory lateralization (with respect to the corresponding pre-TMS blocks) to the right, and positive AMP displacements indicate shifts to the left (Fig. $1 B$ ). This normalization procedure was conducted because subjects usually exhibited a slight bias of the AMP to the left or right in the pre-TMS reference condition. In particular, as is obvious from the leftward shift of the mean pre-TMS AMP in Figure $1 A$, the majority of subjects may have had a preference to press the "right" key in cases in which they perceived the sound to be exactly on the median plane or were uncertain whether it had been to the left or right.

Two additional control experiments were conducted to yield estimates of nonspecific effects of rTMS on sound lateralization. Apart from the locus of rTMS, conditions were as in the main experiment. In one control experiment, rTMS was applied to the occipital cortex $(2 \mathrm{~cm}$ above and 2 $\mathrm{cm}$ lateral of the inion). Stimulation of this cortical area, which is not involved in auditory processing, had no effect on sound lateralization. In the other control experiment, the facial nerve $(2 \mathrm{~cm}$ above and $2 \mathrm{~cm}$ ventral of the tragus) was stimulated, because stimulation of parietal areas leads to a considerable contraction of the ipsilateral facial muscles. No shift in sound lateralization could be recorded in this control experiment (see Fig. 3).

\section{RESULTS}

Subjects made two-alternative forced-choice (left/right) judgements on the intracranial position of sound images, which were evoked by the presentation of dichotic tone pulses with variable ITDs. Four blocks of the task, each lasting 11 min, were conducted, two of them before a $10 \mathrm{~min}$ period of low-frequency rTMS $(1 \mathrm{~Hz})$ of the PPC (reference, pre-TMS blocks 1 and 2), and two identical blocks immediately after rTMS (post-TMS blocks 1 and 2).

In the post-rTMS block 1, the subject's AMP (point of equal numbers of left and right judgements) was, with respect to the pre-TMS block 1, significantly shifted toward the side of rTMS. That is, a sound image, evoked by a constant ITD, appeared more to the left after rTMS of the right PPC $(p=0.010)$ and more to the right after rTMS of the left PPC ( $p=0.027$; Fisher randomization test for matched pairs) (Fig. 1). The mean auditory shift obtained in the first block after rTMS was slightly stronger for stimulation of the right hemisphere $(12.0 \mu \mathrm{sec} ; \mathrm{SEM}, \pm 2.8 \mu \mathrm{sec})$ compared with left rTMS (6.3 $\mu \mathrm{sec}$; SEM, $\pm 3.6 \mu \mathrm{sec})$. However, this tendency did not reach the level of statistical significance. Intersubject variability was relatively large in these experiments: although the maximum shifts obtained after left or right rTMS
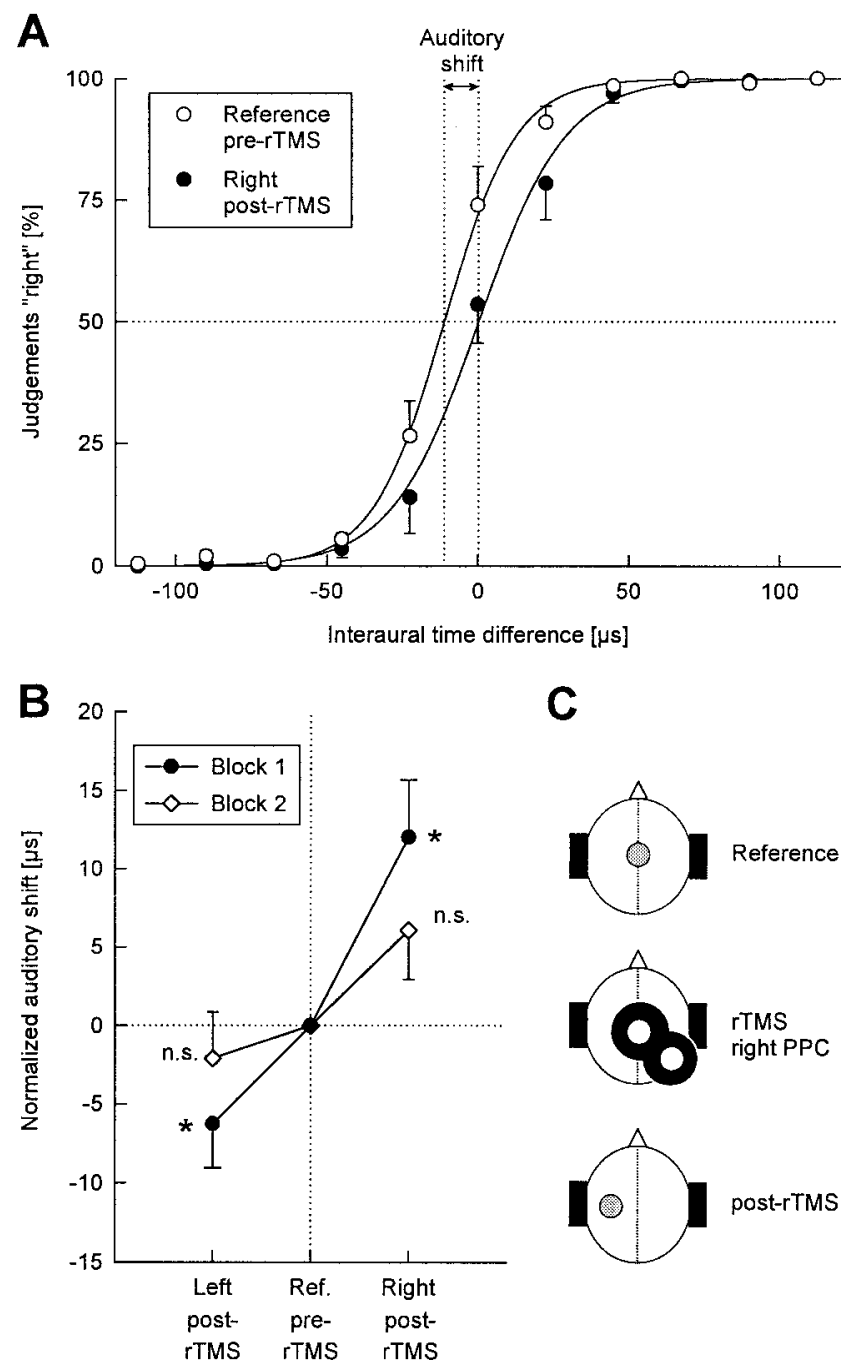

C
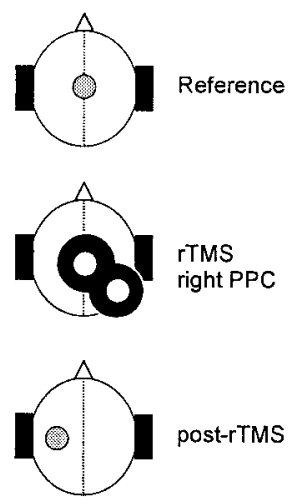

Figure 1. Effects of rTMS of the PPC on lateralization of interaural time differences. $A$, Mean psychometric functions for all subjects before (prerTMS) and after a 10 min period of rTMS of the right PPC (post-rTMS; block 1). Original judgements (symbols, mean \pm SEM) were fitted with sigmoid functions. The AMP of the head was defined as that interaural time difference at which the frequency of left and right judgements was $50 \%$ (indicated by the points of intersection of the dotted lines). B, Mean shift of the AMP for the periods of 0-11 min (Block 1, closed symbols, mean \pm SEM) and 11-22 min (Block 2, open symbols, mean \pm SEM) after rTMS of the left or right PPC. Data are normalized with respect to the pre-rTMS reference condition. Positive values indicate shifts of the AMP to the right or shifts of the auditory percept to the left; negative values are vice versa. Asterisks indicate significant differences between the reference and post-rTMS condition (n.s., nonsignificant). $C$, Schematic illustration of the main effect. An intracranial sound image (gray-shaded area), which was originally centered to the AMP with the reference condition, was perceived as shifted to the left after application of rTMS to the PPC of the right hemisphere.

were more than $\pm 25 \mu \mathrm{sec}$, in some subjects, rTMS appeared to be less efficient, so that auditory shifts were close to zero.

Only insignificant displacements of the AMP were obtained in the post-rTMS block 2, indicating the decrease of the rTMS after-effect as a function of time. As shown in Figure 2, analyses of the psychometric functions revealed no significant changes in the acuity of auditory lateralization after rTMS. Rather, a significant reduction in the mean reaction times of the subjects' judgements after rTMS (block 1; right rTMS, -39.3 msec; left rTMS, 

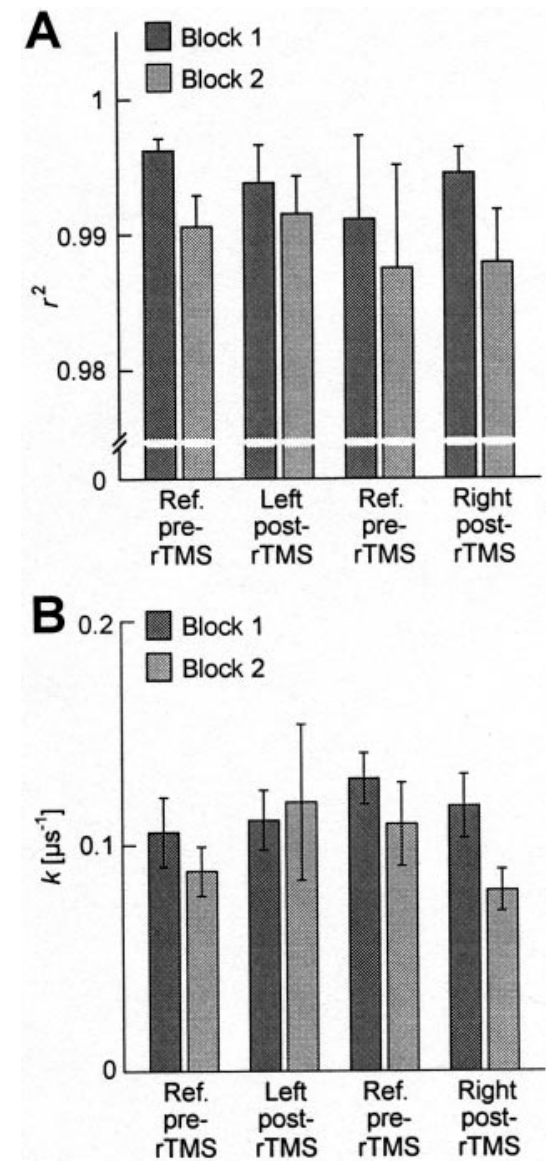

Figure 2. Acuity of the discrimination of interaural time differences. $A$, Mean coefficients of determination $\left(r^{2}\right.$, mean \pm SEM) resulting from the fit of individual data to a sigmoid function. $B$, Mean slopes $(k)$ of the individual fitted functions. Dark gray columns indicate mean values (mean \pm SEM) for block 1 of the pre-rTMS or post-rTMS conditions; light gray columns indicate values for block 2. Differences between corresponding pre-rTMS and post-rTMS data are nonsignificant in each case.

$-35.1 \mathrm{msec} ; p<0.0001$ in both cases; $t$ test) was found, although subjects were instructed merely to respond within $1 \mathrm{sec}$ after presentation of the sound stimulus. Because this latter effect was also observed in control experiments using rTMS of the occipital cortex, it is likely caused by an enhancement of general vigilance. Apart from this effect, control experiments with application of rTMS to either the occipital cortex or the facial nerve gave no hint of nonspecific effects of rTMS on the subject's performance. This can be taken as evidence that the effects of rTMS to the PPC are indeed specific (Fig. 3).

\section{DISCUSSION}

The primary finding of the present study is that focal magnetic stimulation of the right or left PPC results in a systematic shift of sound lateralization but does not affect the precision of ITD discrimination. Because the ITD is a main cue for sound azimuth, this auditory shift may correspond to an azimuthal shift of auditory space. Thus, the PPC may play a specific role in the localization of absolute sound-source positions but may not be involved in discrimination of the relative positions of different sound sources.

The direction of the auditory shift toward the side contralateral to rTMS application (Fig. 1C) can be explained on the basis of two considerations. First, previous studies that have used rTMS at low frequencies have suggested an inhibitory rather than an

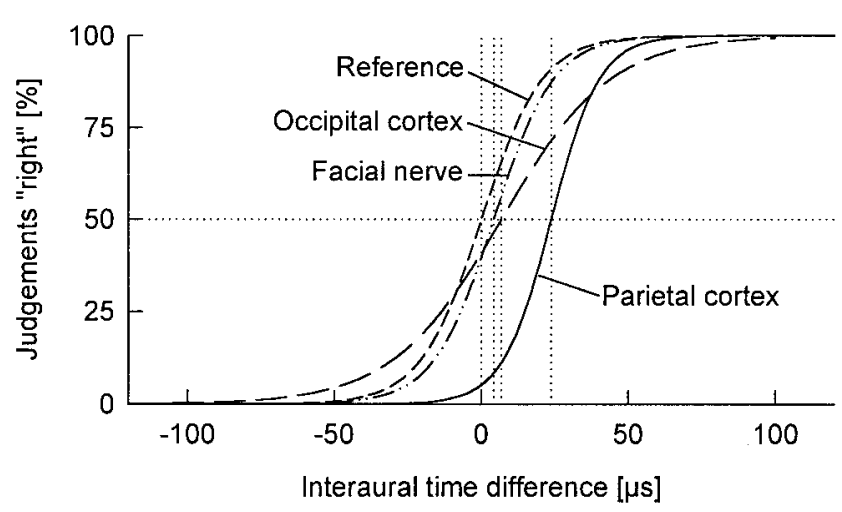

Figure 3. Individual psychometric functions of one subject obtained in the main experiment (rTMS of the posterior parietal cortex) and in two control experiments with rTMS of the occipital cortex or of the facial nerve. Fitted functions are computed from block 1 data with the pre-rTMS reference conditions or after right-hemispheric rTMS and are normalized with respect to the pre-rTMS curve. The positions of the auditory median plane are indicated by the points of intersection of the dotted lines. Data are from a subject who exhibited relatively large auditory shifts in the main experiment (compare Fig. $1 A$ ). Apart from that fact, the psychometric functions of the other subjects were similar to those shown here.

activating influence on neural processing in the underlying cortical surface (Chen et al., 1997). Second, neurophysiological recordings of spatially selective auditory neurons in the monkey parietal cortex have demonstrated preferential tuning to sounds located in the contralateral hemispace (Mazzoni et al., 1996; Stricanne et al., 1996). Thus, the present auditory shift may not be induced by mere inhibition of auditory afferents from the contralateral ear, which would have resulted in an opposite shift. A more likely interpretation is that rTMS has specifically affected processes of neural coordinate transformations, such as those recently demonstrated in the monkey parietal cortex for the visual domain. In the monkey lateral intraparietal area, visual receptive fields of single neurons have been shown to be referenced to the body (Brotchie et al., 1995), and a world-referenced coding of visual space was found in the adjacent parietal area 7a (Snyder et al., 1998). Although there is as of yet no direct experimental evidence, it seems reasonable to assume that this also holds true for the auditory modality: a substantial proportion of monkey parietal neurons is known to have a spatial selectivity not only for visual but also for acoustic stimuli (Mazzoni et al., 1996; Stricanne et al., 1996). Assuming homology with the monkey parietal cortex, auditory space may thus be transformed in the human PPC from the originally head-centered into body- and/or worldcentered coordinates. That is to say, when the head is rotated under normal conditions to, for example, the right, auditory receptive fields in the PPC may systematically shift, with respect to the head, toward the left, in a manner such that they remain stationary with respect to external space. Inhibition of the PPC of one hemisphere by rTMS should thus result in an interhemispheric imbalance of this neural mechanism of coordinate transformation, as may, under normal conditions, occur with body motion. That is, the cortical representation of auditory space may be biased toward the nonstimulated, contralateral hemisphere. A perceptual correlate of this bias may be that the auditory spatial frame of reference, namely the AMP, is displaced toward the ipsilateral hemispace. Consequently, sounds physically centered to the median sagittal plane should be heard displaced to the side contralateral to rTMS, as was the case in the present experiment. 
Coordinate transformations such as those hypothesized above must integrate auditory spatial information with head-to-trunk position and vestibular (head-in-space position) signals. In this context, it is interesting that an auditory shift that is virtually identical to that demonstrated here after rTMS of, for example, the right PPC can be also found when the subject's head is oriented to the right (Lewald and Ehrenstein, 1998), when the muscle spindles of the left neck muscles are stimulated by vibration (Lewald et al., 1999), during rotatory acceleration of the subject's whole body to the left (Lewald and Karnath, 2001), and after right-sided, cold-caloric vestibular stimulation (Lewald and Karnath, 2000). In other words, direct inhibition of the PPC of one hemisphere by rTMS may be equivalent to (1) neckproprioceptive afferent signals, conveying head positions to the ipsilateral side, or (2) vestibular afferent signals, conveying rotations to the contralateral side. These similarities further support the view that rTMS of the PPC may have specifically affected neural coordinate transformations, integrating auditory with neck-proprioceptive and vestibular inputs.

In obvious alignment with our findings, previous neuroimaging studies have demonstrated bilateral activation of the PPC with sound-localization tasks (Griffiths et al., 1998; Bushara et al., 1999; Weeks et al., 1999). The pronounced right-hemispheric predominance obtained in those studies may correspond to the present observation that the mean auditory shift was larger after right rTMS than after left rTMS (Fig. 1B). This tendency, although statistically nonsignificant, is in accord with the view of a preferential role of the right PPC for sound localization.

Previous studies on patients with brain lesions including the PPC have also reported shifts in auditory localization or lateralization compared with healthy subjects. The majority of those studies, although not all (Vallar et al., 1995), have demonstrated more or less pronounced systematic errors toward the right after lesions of the right hemisphere, and either nonsignificant or dispersed errors after left-hemispheric lesions (Bisiach et al., 1984; Pinek et al., 1989; Pinek and Brouchon, 1992; Tanaka et al., 1999; Bellmann et al., 2001). In general, this seems to be in agreement with the present results. However, the directions of the auditory shifts found after rTMS and after brain lesions are apparently opposite if rTMS is supposed to induce transient "virtual lesions" (Walsh and Cowey, 2000). One must nevertheless consider that actual brain damage usually involves larger parts of several cortical and subcortical brain regions, so that conclusions on the specific role of the PPC are difficult to draw. A critical point in the studies mentioned above may be that most patients suffered from a hemispatial neglect (a lack of awareness of space on the side of the body contralateral to a brain injury). This disorder has been attributed recently to lesions of the right superior temporal cortex (Karnath, 2001; Karnath et al., 2001), which is known to be involved in neural processing of auditory space (Rauschecker, 1998; Romanski et al., 1999; Rauschecker and Tian, 2000; Tian et al., 2001). But whereas absolute localization may be the domain of the parietal cortex in spatial hearing, the human right superior temporal cortex may rather be important for auditory spatial discriminative abilities (Zimmer et al., 2001).

In contrast to studies on patients with brain lesions, the present method may have reversibly affected a relatively restricted area of intact cortex, so that our results may be more reliable to interpret than those obtained from brain-damaged patients. Another major difference between the "virtual", short-lasting lesions induced by
TMS and structural lesions is the fact that compensatory mechanisms do not play a role in TMS-induced lesions. In conclusion, the present study suggests that the human PPC represents the neural substrate of transformations of auditory spatial coordinates that may be the prerequisite for the perceptual stability of auditory (and multisensory) space during motion (Thier and Karnath, 1997).

\section{REFERENCES}

Bellmann A, Meuli R, Clarke S (2001) Two types of auditory neglect. Brain 124:676-687.

Bisiach E, Cornacchia L, Sterzi R, Vallar G (1984) Disorders of perceived auditory lateralization after lesions of the right hemisphere. Brain 107:37-52.

Brotchie PR, Andersen RA, Snyder LH, Goodman SJ (1995) Head position signals used by parietal neurons to encode locations of visual stimuli. Nature 375:232-235.

Bushara KO, Weeks RA, Ishii K, Catalan M-J, Tian B, Rauschecker JP, Hallett M (1999) Modality-specific frontal and parietal areas for auditory and visual spatial localization in humans. Nat Neurosci 2:759-766.

Chen R, Classen J, Gerloff C, Celnik P, Wassermann EM, Hallett M, Cohen LG (1997) Depression of motor cortex excitability by lowfrequency transcranial magnetic stimulation. Neurology 48:1398-1403.

Griffiths TD, Rees G, Rees A, Green GGR, Witton C, Rowe D, Büchel C, Turner R, Frackowiak RSJ (1998) Right parietal cortex is involved in the perception of sound movement in humans. Nat Neurosci 1:74-79.

Hallett M (2000) Transcranial magnetic stimulation and the human brain. Nature 406:147-150.

Karnath H-O (2001) New insights into the functions of the superior temporal cortex. Nat Rev Neurosci 2:568-576.

Karnath H-O, Ferber S, Himmelbach M (2001) Spatial awareness is a function of the temporal not the posterior parietal lobe. Nature 411:950-953.

Lewald J, Ehrenstein WH (1998) Influence of head-to-trunk position on sound lateralization. Exp Brain Res 121:230-238.

Lewald J, Karnath H-O (2000) Vestibular influence on human auditory space perception. J Neurophysiol 84:1107-1111.

Lewald J, Karnath H-O (2001) Sound lateralization during whole-body rotation. Eur J Neurosci 13:2268-2272.

Lewald J, Karnath H-O, Ehrenstein WH (1999) Neck-proprioceptive influence on auditory lateralization. Exp Brain Res 125:389-396.

Masterton RB (1992) Role of the central auditory system in hearing: the new direction. Trends Neurosci 15:280-285.

Mazzoni P, Bracewell RM, Barash S, Andersen RA (1996) Spatially tuned auditory responses in area LIP of macaques performing delayed memory saccades to acoustic targets. J Neurophysiol 75:1233-1241.

Middlebrooks JC (2000) Cortical representations of auditory space. In: The new cognitive neurosciences (Gazzaniga MS, ed), pp 425-436. Cambridge, MA: MIT.

Mishkin M, Ungerleider LG, Macko KA (1983) Object vision and spatial vision: two cortical pathways. Trends Neurosci 6:414-417.

Mottaghy FM, Hungs M, Brügmann M, Sparing R, Boroojerdi B, Foltys H, Huber W, Töpper R (1999) Facilitation of picture naming after repetitive transcranial magnetic stimulation. Neurology 53:1806-1812.

Pascual-Leone A, Valls-Sole J, Wassermann EM, Hallett M (1994) Responses to rapid-rate transcranial magnetic stimulation of the human motor cortex. Brain 117:847-858.

Pinek B, Brouchon M (1992) Head turning versus manual pointing to auditory targets in normal subjects and in subjects with right parietal damage. Brain Cogn 18:1-11.

Pinek B, Duhamel J-R, Cavé C, Brouchon M (1989) Audio-spatial deficits in humans: differential effects associated with left versus right hemisphere parietal damage. Cortex 25:175-186.

Rauschecker JP (1998) Cortical processing of complex sounds. Curr Opin Neurobiol 8:516-521.

Rauschecker JP, Tian B (2000) Mechanisms and streams for processing of "what" and "where" in auditory cortex. Proc Natl Acad Sci USA 97:11800-11806.

Romanski LM, Tian B, Fritz J, Mishkin M, Goldman-Rakic PS, Rauschecker JP (1999) Dual streams of auditory afferents target multiple domains in the primate prefrontal cortex. Nat Neurosci 2:1131-1136.

Snyder LH, Grieve KL, Brotchie P, Andersen RA (1998) Separate body- and world-referenced representations of visual space in parietal cortex. Nature 394:887-891.

Stricanne B, Andersen RA, Mazzoni P (1996) Eye-centered, headcentered, and intermediate coding of remembered sound locations in area LIP. J Neurophysiol 76:2071-2077.

Tanaka H, Hachisuka K, Ogata HJ (1999) Sound lateralisation in patients with left or right cerebral hemispheric lesions: relation with unilateral visuospatial neglect. J Neurol Neurosurg Psychiatry 67:481-486. 
Thier P, Karnath H-O (1997) Parietal lobe contributions to orientation in 3D space. Heidelberg: Springer.

Tian B, Reser D, Durham A, Kustov A, Rauschecker JP (2001) Functional specialization in rhesus monkey auditory cortex. Science 292:290-293.

Töpper R, Foltys H, Mottaghy FM, Boroojerdi B (1999) Repetitive transcranial magnetic stimulation of the parietal cortex influences motor imagery. Electroencephalogr Clin Neurophysiol Suppl 51:145-150.

Vallar G, Guariglia C, Nico D, Bisiach E (1995) Spatial hemineglect in back space. Brain 118:467-472.

Walsh V, Cowey A (2000) Transcranial magnetic stimulation and cognitive neuroscience. Nat Rev Neurosci 1:73-79.
Wassermann EM (1998) Risk and safety of repetitive transcranial magnetic stimulation: report and suggested guidelines from the International Workshop on the Safety of Repetitive Transcranial Magnetic Stimulation, June 5-7, 1996. Electroencephalogr Clin Neurophysiol 108:1-16.

Weeks RA, Aziz-Sultan A, Bushara KO, Tian B, Wessinger CM, Dang N, Rauschecker JP, Hallett M (1999) A PET study of human auditory spatial processing. Neurosci Lett 262:155-158.

Zimmer U, Lewald J, Karnath H-O (2001) Disturbed lateralization of dichotic sound in patients with neglect. In: Göttingen Neurobiology Report 2001: Proceedings of the 2nd Göttingen Conference of the German Neuroscience Society 2001, Vol 2 (Elsner N, Kreutzberg GW, eds), pp 404. Stuttgart, Germany: Thieme. 